\title{
Design rules for strong electro-optic materials
}

\author{
Ali K. Hamze $\mathbb{D}^{1}$, Marc Reynaud ${ }^{1}$, Jacqueline Geler-Kremer ${ }^{1}$ and Alexander A. Demkov $\mathbb{D}^{1 凶}$
}

The explosive rise of silicon photonics has led to renewed interest in the electro-optic (EO) or Pockels effect due to its potential uses in many next generation device applications. To find materials with a strong EO response in thin film form, which are essential for low power and small footprint devices, one needs to find a general design rule for strong Pockels materials. To elucidate what makes the Pockels effect strong, we study the effect in $\mathrm{LiB}_{3} \mathrm{O}_{5}(\mathrm{LBO})$ and $\mathrm{CsB}_{3} \mathrm{O}_{5}(\mathrm{CBO})$ and use these materials as prototypical examples of where conventional wisdom breaks down. We find the Pockels tensor components to be extremely small in both materials, despite the large degree of anharmonicity in the crystals, which has been used as a proxy for the presence of nonlinear electronic effects. We relate the lack of EO response to the large optical phonon frequencies (despite the relatively large Raman susceptibility) in LBO and to the small Raman susceptibility (despite the low phonon frequencies) in CBO, respectively. We shed light on the underlying physical phenomena behind the Raman susceptibility, which we find to be intimately linked to the electron-phonon coupling strength of the near-edge electronic states, and identify a route to discovering new strong EO materials.

npj Computational Materials (2020)6:130; https://doi.org/10.1038/s41524-020-00399-z

\section{INTRODUCTION}

The remarkable ability of a noncentrosymmetric crystal to change its index of refraction in response to an applied electric field known as the Pockels effect ${ }^{1}$, has been the subject of increasing study in recent years due to its potential to facilitate high-speed, low-power electro-optical modulation in integrated photonics applications ${ }^{2-6}$, including intrachip data transmission, neuromorphic logic optical chips $^{7}$, and photonic integrated circuits for quantum computing ${ }^{8,9}$. Being linear in the field, the Pockels, or linear electro-optic (EO), effect coexists with the much smaller Kerr effect $^{10}$ (which is the second order in the field) and with second harmonic generation (SHG). The Pockels effect is most commonly used for optical modulation in the telecommunications industry, where $\mathrm{LiNbO}_{3}$ (LNO), with an unclamped Pockels coefficient of $r_{51}=33 \mathrm{pm} / \mathrm{N}^{11}$, is the current "gold standard" material ${ }^{1,12,13}$. Due to the complexity of integrating $\mathrm{LNO}^{14-17}$ on $\mathrm{Si}$, however, perovskite titanates have recently become the primary subjects of both theoretical $^{18-22}$ and experimental ${ }^{7,17,23-32}$ studies on the use of the Pockels effect in Si photonics. The main focus of these efforts has been $\mathrm{BaTiO}_{3}$ (BTO), due to its integrability with $\mathrm{Si}$ $(001)^{27,28,33}$ and its very large unclamped Pockels effect $\left(r_{42}=\right.$ $\left.1300 \mathrm{pm} / \mathrm{V}^{34}\right)$, which can retain bulk-like values even in thin films ${ }^{7}$.

It is of paramount importance to find materials with large Pockels responses in order to decrease the power consumption and/or size of integrated EO devices. However, because it is not currently clear what mechanism drives the extremely high response of BTO as compared to other materials, the search for potentially better alternatives has so far proceeded rather empirically.

Recent theoretical studies of the Pockels effect in perovskite titanates $^{18-20}$ offer a systematic way forward. Fredrickson et al. ${ }^{19}$ explored strain engineering in BTO. They found critical biaxial strains at which the Pockels response diverged and specific phonon modes went soft. Hamze and Demkov ${ }^{18}$ studied the same phenomenon in strained $\mathrm{SrTiO}_{3}$ (STO), and through the analyses of mode Grüneisen parameters related anharmonicity (soft modes) to an emergent EO response ${ }^{35,36}$. Paillard et al. ${ }^{20}$ reported similar behavior in the EO response of strained $\mathrm{PbTiO}_{3}$ (PTO). These crystals all have in common soft phonons and large crystal anharmonicity. A 1-D anharmonic oscillator model of the Pockels effect $^{37}$ shows the Pockels parameter is directly proportional to the coefficient of the anharmonic force term, reinforcing the assumed relationship between anharmonicity and the EO response. Noticing that, in these materials, the Pockels effect is dominated by the lattice, the appropriate figure of merit is the ratio of the Raman susceptibility and square of the phonon frequency. One may therefore infer the following design rule: highly anharmonic, noncentrosymmetric crystals should exhibit a large Pockels effect.

Crystal anharmonicity is often associated with large thermal expansion coefficients. In Table 1 , we list a selection of noncentrosymmetric crystals and their material properties. BTO, STO, and PTO all have large thermal expansion coefficients, as do many other strong Pockels materials. Therefore, an ideal candidate to test the proposed design rule is $\mathrm{LiB}_{3} \mathrm{O}_{5}(\mathrm{LBO})$. Though there are no reported Pockels measurements of LBO (Fig. 1), it is a commonly used nonlinear optical crystal ${ }^{38}$ with many favorable optical properties ${ }^{39,40}$ and it boasts a thermal expansion coefficient an order of magnitude larger ${ }^{41,42}$ than that of BTO, STO, or PTO.

In this article, using first principles calculations, we set out to identify the microscopic origin of a material's large Pockels response. Our calculations have uncovered a deeper, more complex physics than previously demonstrated. Contrary to our initial expectations, LBO has a very weak Pockels response due to the low atomic mass of Li leading to high phonon frequencies. We attempt to induce a stronger response by softening the modes through replacement of the light $\mathrm{Li}$ atoms with much heavier $\mathrm{Cs}$ atoms by considering the related $\mathrm{CsB}_{3} \mathrm{O}_{5}$ (CBO) crystal. This successfully softens the phonon modes but does not improve the Pockels response, thereby highlighting the importance of a different property, the Raman susceptibility, a connection originally pointed out by Johnston and Kaminow ${ }^{43,44}$. We contrast our results for the $\mathrm{LBO}$ and CBO to previous work on strained STO

\footnotetext{
${ }^{1}$ Department of Physics, The University of Texas at Austin, Austin, TX 78712, USA. ${ }^{\circledR}$ email: demkov@physics.utexas.edu
} 
Table 1. Physical properties of known EO materials.

\begin{tabular}{|c|c|c|c|c|c|c|c|c|c|c|}
\hline Crystal & Space group & $a\left(10^{-6}{ }^{\circ} \mathrm{C}^{-1}\right)$ & $T\left({ }^{\circ} \mathrm{C}\right)$ & $r(\mathrm{pm} / \mathrm{V})$ & $n$ & $\lambda(\mathrm{nm})$ & $n^{3} r(p m / V)$ & $E_{g}(\mathrm{eV})$ & Sources & Notes \\
\hline $\mathrm{Bi}_{12} \mathrm{GeO}_{20}$ & 123 & 15 & 20 & $r_{41}^{\sigma}=3.22$ & 2.54 & 666 & 53 & 3.2 & $70-73$ & $(1938283)$ \\
\hline $\mathrm{LiNbO}_{3}$ & $R 3 c$ & 15.9 & $0-500$ & $r_{51}^{\sigma}=33$ & 2.286 & 633 & 389 & 4.9 & $11,72,76-78$ & (543592) \\
\hline $\mathrm{LiTaO}_{3}$ & $R 3 c$ & 16.2 & $0-500$ & $r_{33}^{\sigma}=30.5$ & 2.18 & 632.8 & 316 & $3.41-4.56$ & $10,78-80$ & (553579) \\
\hline $\mathrm{BaTiO}_{3}$ & P4mm & 16.9 & 25 & $r_{42}^{\sigma}=1300$ & 2.45 & 546 & 24,118 & 3.2 & $34,72,81-83$ & (1925967) \\
\hline $\mathrm{Ba}_{0.25} \mathrm{Sr}_{0.75} \mathrm{TiO}_{3}$ & P4bm & 12.7 & $0-90$ & $r_{\text {eff }}^{\sigma}=844$ & 2.37 & 510 & 11,235 & 3.88 & $1,85-87,89-91$ & - \\
\hline $\mathrm{KNbO}_{3}$ & $A m m 2$ & 14 & $20-180$ & $r_{42}^{\sigma}=450$ & 2.329 & 633 & 4801 & $3.43-3.6$ & $1,92-94$ & (1923882) \\
\hline $\mathrm{MgO}: \mathrm{LiNbO}_{3}$ & $R 3 c$ & - & - & - & 2.39 & 355 & - & 3.92 & 95,96 & (1414411) \\
\hline$\beta-\mathrm{BaB}_{2} \mathrm{O}_{3}$ & $R 3 c$ & 45 & $20-700$ & $r_{22}^{\sigma}=2.5$ & 1.67 & 514 & 12 & 6.43 & 97-99 & $(556972)$ \\
\hline $\mathrm{LiB}_{3} \mathrm{O}_{5}$ & Pna $_{1}$ & 108.2 & $17-790$ & - & 1.68 & 253.7 & - & 7.78 & $39,42,97,98$ & $(311713)$ \\
\hline $\mathrm{Pb}\left[\mathrm{Zr}_{x} \mathrm{Ti}_{1-x}\right] \mathrm{O}_{3}$ & $P 4 m m, R 3 c$ & 8 & 527 & $r_{\mathrm{eff}}^{\sigma}=109$ & 2.45 & 633 & 1603 & $3.4-3.6$ & 1,109-113 & $\begin{array}{l}\text { Tetragonal: (380620); Trigonal: } \\
(1228661) ; n, r_{\mathrm{eff}}^{\sigma}, E_{g}: x=0.4 ; \\
a: x=0.19\end{array}$ \\
\hline $\mathrm{BeO}$ & $P 6_{3} m c$ & 10.57 & 727 & - & 1.74 & 440 & - & 10.6 & 114-117 & $(1500435)$ \\
\hline$\kappa-\mathrm{Al}_{2} \mathrm{O}_{3}$ & $\mathrm{Pna}_{1}$ & 8.1 & 840 & - & 2.5 & 124 & - & 5.4 & 118,119 & - \\
\hline $\begin{array}{l}\mathrm{P}_{1-x} \mathrm{La}_{x}\left(\mathrm{Zr}_{y} \mathrm{Ti}_{z}\right)_{1} \\
-x / 4 \mathrm{O}_{3}\end{array}$ & $P 4 m m, R 3 c$ & $5.7^{*}$ & 25 & $r_{\text {eff }}^{\sigma}=153$ & $2.64 \wedge$ & 633 & 2815 & 3.5 & $1,120-124$ & $\begin{array}{l}\wedge x, y, z=0.08,0.1,0.9: \text { trigonal } \\
(1222146) ;{ }^{*} x, y, z=0.07,0.65 \\
0.35: \text { tetragonal } \\
(1141504) ; E_{g}: x, y, z=0.05 \\
0.54,0.46\end{array}$ \\
\hline
\end{tabular}

Physical properties of many crystals known to have a linear EO response and the conditions under which they were measured, as well as some nonlinear crystals with unknown Pockels coefficients. All of them have noncentrosymmetric space groups (a necessary condition for a nonzero EO response), and all of them are highly nonlinear (as compared to silicon, which is highly linear and has $a=2.93 \times 10^{-6}{ }^{\circ} \mathrm{C}^{125}$ ), especially those with very high EO response. LiB $\mathrm{O}_{5}$ stands out with $a=108.2 \times 10^{-6}{ }^{\circ} \mathrm{C}$, a full order of magnitude larger than any other crystal on the table. Space groups of the crystals are from Pearson's Crystal Database, and the seven-digit numbers in parentheses in the Notes column are the reference numbers of specific entries in the database ${ }^{126}$. Thermal expansion coefficients listed are the largest ones found for the given materials. Refractive indices and wavelengths are either values that closely coincide with $r_{\text {eff }}$ if $r_{\text {eff }}$ is specified, or are the largest values found. Effective Pockels coefficients, labeled $r_{\text {eff, }}$, were measured in thin film materials. Where reported, we labeled whether the measured Pockels coefficient is clamped or unclamped.

The symbols point towards to the Notes column, where additional pieces of information for the measurement marked is presented.

and, using a simple tight-binding model, associate large Raman susceptibilities with the deformation potential of the material. Now, our refined design rule states that in addition to the absence of inversion symmetry, a large Raman susceptibility (strong electron-phonon coupling) and soft phonons are essential ingredients to produce a large EO response.

\section{RESULTS}

Theoretical framework

All calculations are performed within density functional theory (DFT) using ABINIT ${ }^{45-51}$ and follow the theoretical framework developed by Veithen et al. $^{49}$ (see the "Methods" section for details). Macroscopically, the Pockels coefficient $r$ relates the change in the index of refraction to an applied electric field as $n(E)=n_{0}-\frac{1}{2} r n_{0}^{3} E$, where $n_{0}$ is the index of refraction under no field ${ }^{1}$. In a crystal, a modulating electric field can couple directly to the electrons, to the lattice, or can cause a lattice modulation via the inverse piezoelectric effect. Therefore, in general, one can write the change of the dielectric tensor in response to the external field $a s^{52}$

$\frac{d \epsilon_{i j}}{d E_{k}}=\sum_{m n} \frac{\partial \epsilon_{i j}}{\partial \pi_{m n}} \frac{\partial \pi_{m n}}{\partial E_{k}}+\sum_{l} \frac{\partial \epsilon_{i j}}{\partial P_{l}} \frac{\partial P_{l}}{\partial E_{k}}+\frac{\partial \epsilon_{i j}}{\partial E_{k}}$.
Here, $\pi$ is strain and $P$ is the ionic polarization. The last term is the direct effect of the applied field on the polarizability, and only electrons can respond at the optical probing frequency; this is SHG $\left(x^{(2)}\right)$. The first term describes the effect of strain caused by the applied field and is present at low modulating frequencies-this is the unclamped response, meaning the lattice has time to deform in response to the applied field, and it can be neglected at high modulating frequencies. The second term is the so-called ionic or lattice contribution to the EO response and is at the center of our study.

Microscopically, the Pockels coefficient is a tensor $r_{i j k}$ that relates the change in the ij component of the inverse dielectric tensor of a noncentrosymmetric crystal to a static, external electric field applied in the $k_{\text {-direction }}{ }^{10,49,53}$

$\Delta\left(\epsilon^{-1}\right)_{i j}=\sum_{k} r_{i j k} E_{k}$

There are three contributions to the Pockels tensor: an electronic component, an ionic component, and a piezoelectric component due to the converse piezoelectric effect ${ }^{49}$. These three 
(a)

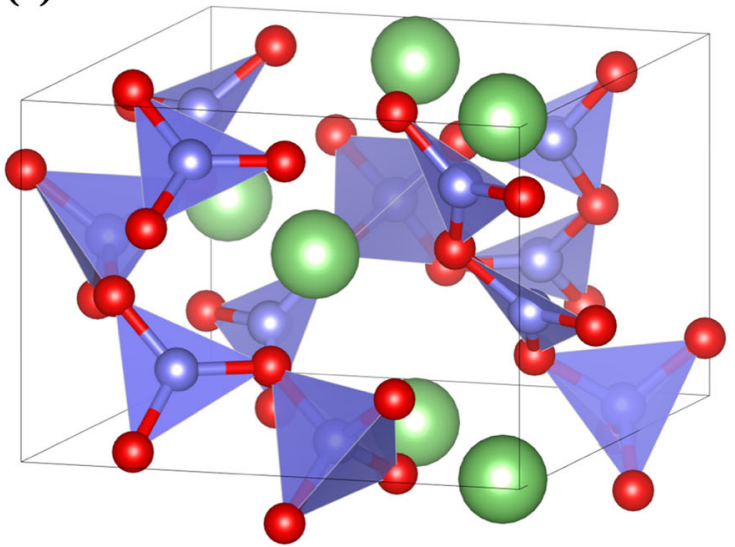

(b)

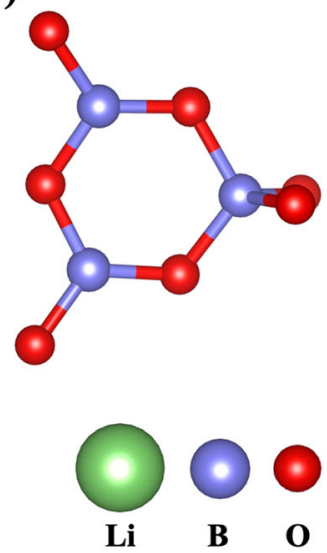

Fig. 1 LiB305 crystal structure. The crystal structure of LBO. Its space group is Pna2 1 and its structure ${ }^{41,127,128}$ consists of a helical network of $\mathrm{B}_{3} \mathrm{O}_{7}$ rings that share one of their oxygen atoms with neighboring $\mathrm{B}_{3} \mathrm{O}_{7}$ groups. Two of the $\mathrm{B}$ atoms threefold coordinated and one is fourfold coordinated. The $\mathrm{Li}$ atoms reside in the voids of the $\mathrm{B}_{3} \mathrm{O}_{7}$ helices, which are along the $c$-axis. a Unit cell of $\mathrm{LiB}_{3} \mathrm{O}_{5}\left(\right.$ at room temperature ${ }^{41}$ ), and b the $\mathrm{B}_{3} \mathrm{O}_{7}$ rings that make up the helical chains.

components are given by (in the principal axes of the crystal) ${ }^{49}$

$r_{i j k}^{\text {piezo }}=\sum_{\mu, \nu=1}^{3} p_{i j \mu \nu} d_{k \mu \nu}$,

$r_{i j k}^{\text {ion }}=-\frac{4 \pi}{\sqrt{\Omega_{0}} n_{i}^{2} n_{j}^{2}} \sum_{m} \frac{a_{i j}^{m} p_{m, k}}{\omega_{m}^{2}}$

$r_{i j k}^{\mathrm{el}}=-\left.\frac{8 \pi}{n_{i}^{2} n_{j}^{2}} x_{i j l}^{(2)}\right|_{l=k}$,

where $n$ is the index of refraction, $X_{i j l}^{(2)}$ is the nonlinear optical susceptibility, $\Omega_{0}$ is the volume of the unit cell, and $a_{i j}^{m}, p_{m, k}$, and $\omega_{m}$ are the Raman susceptibility, the mode polarity, and the frequency of the mode $m$ at the $\Gamma$ point, respectively, $p_{i j \mu \nu}$ is the elasto-optic (photoelastic) tensor, and $d_{k \mu \nu}$ is the piezoelectric tensor. Equations (3-5) are the microscopic forms of the first, second, and third terms in Eq. (1), respectively. The Raman susceptibility and mode polarity are given by

$a_{i j}^{m}=\sqrt{\Omega_{0}} \sum_{\gamma, \beta} \frac{\partial X_{i j}^{(1)}}{\partial \tau_{\gamma \beta}} u_{m}(\gamma \beta)$,

$p_{m, k}=\sum_{\gamma, \beta} Z_{\gamma, k \beta}^{*} u_{m}(\gamma \beta)$,

where $\gamma$ labels an atom, $\beta$ labels a direction, $\tau_{\gamma \beta}$ is a displacement of atom $\gamma$ in direction $\beta, X_{i j}^{(1)}$ is the electronic dielectric susceptibility tensor, $Z_{\gamma, k \beta}^{*}$ is the Born effective charge, and $u_{m}(\gamma \beta)$ is the eigendisplacement of atom $\gamma$ in direction $\beta$ in mode $m$ (which relates to the eigenvector by a factor of $1 / \sqrt{M_{\gamma}}$, where $M_{\gamma}$ is the mass of atom $\gamma$ ). The total Pockels tensor is grouped into two parts, a strain-free (clamped) part and a stress-free (unclamped) part. The clamped Pockels tensor $r_{i j k}^{\eta}$ is the sum of the electronic and ionic contributions, and the unclamped tensor $r_{i j k}^{\sigma}$ adds the piezoelectric contribution

$r_{i j k}^{\eta}=r_{i j k}^{\mathrm{el}}+r_{i j k}^{\mathrm{ion}}$

$r_{i j k}^{\sigma}=r_{i j k}^{\eta}+r_{i j k}^{\text {piezo }}$.

Note that we use Voigt notation ${ }^{10}$ to collapse the first two indices when reporting our predictions of the Pockels tensor.

In our analysis of the crystal anharmonicity, we make use of isotropic mode Grüneisen parameters ${ }^{35}$ (Eq. (S1))-a brief overview of how to compute them is provided in the Supplementary information. Finally, we also calculate deformation potentials ${ }^{54-56}$, which give a measure of the electron-phonon coupling, for select phonon modes. The deformation potential $d_{m}$ for optical phonon $m$ is defined by the change in the energy of the conduction band minimum (CBM) $E_{C}$ under displacements of the ionic positions according to the phonon mode $m$ with amplitude $\delta_{m}$

$d_{m}^{c}=\frac{\partial E_{C}}{\partial \delta_{m}}$.

Therefore, once the deformation potential is known, the change of the CBM can be calculated for a given phonon amplitude as $\Delta E_{C}=d_{m} \delta_{m}$. An analogous expression can be defined for the valence band maximum (VBM), which can then be added to the expression for the CBM to give the change in the band gap. However, because we set the VBM to zero in our calculations, we only consider the CBM. The larger the deformation potentials for the CBM and VBM are for a given phonon mode, the larger the change in the band gap under atomic displacements.

\section{LBO and CBO Pockels tensors}

The five largest components of the Pockels tensor for LBO, broken into electronic, ionic, and piezoelectric contributions, are listed in Table 2 . The predicted values are extremely small.

Comparing the components, we see that the ionic contribution is of the same order as the electronic and piezoelectric contributions, despite the extraordinary anharmonicity of the LBO crystal. Recall from Eq. (4), however, that the ionic contribution is inversely proportional to phonon frequencies at $\Gamma$, squared. The large phonon frequencies in LBO (Table S1), an order of magnitude larger than those in perovskite titanates ${ }^{18-20}$, suppress the EO response. How do these high phonon frequencies coexist with the large thermal anharmonicity? A high-temperature 
Table 2. EO tensor components of LBO, CBO, and strained STO.

\begin{tabular}{|c|c|c|c|c|c|}
\hline Crystal & EO tensor component & $r^{\mathrm{el}}(\mathrm{pm} / \mathrm{V})$ & $r^{\text {ion }}(\mathrm{pm} / \mathrm{V})$ & $r^{\text {piezo }}(\mathrm{pm} / \mathrm{V})$ & $r^{\sigma}(\mathrm{pm} / \mathrm{V})$ \\
\hline \multirow{3}{*}{ LBO } & $r_{51}$ & 0.484 & -0.367 & -0.330 & -0.213 \\
\hline & $r_{23}$ & -0.563 & 0.391 & 0.190 & 0.017 \\
\hline & $r_{33}$ & 0.083 & -0.108 & 2.505 & 2.480 \\
\hline CBO & $r_{52}$ & 0.928 & 0.069 & - & - \\
\hline STO (-1.2\%) & $r_{33}$ & -0.085 & -236.469 & -28.90 & -265.454 \\
\hline
\end{tabular}
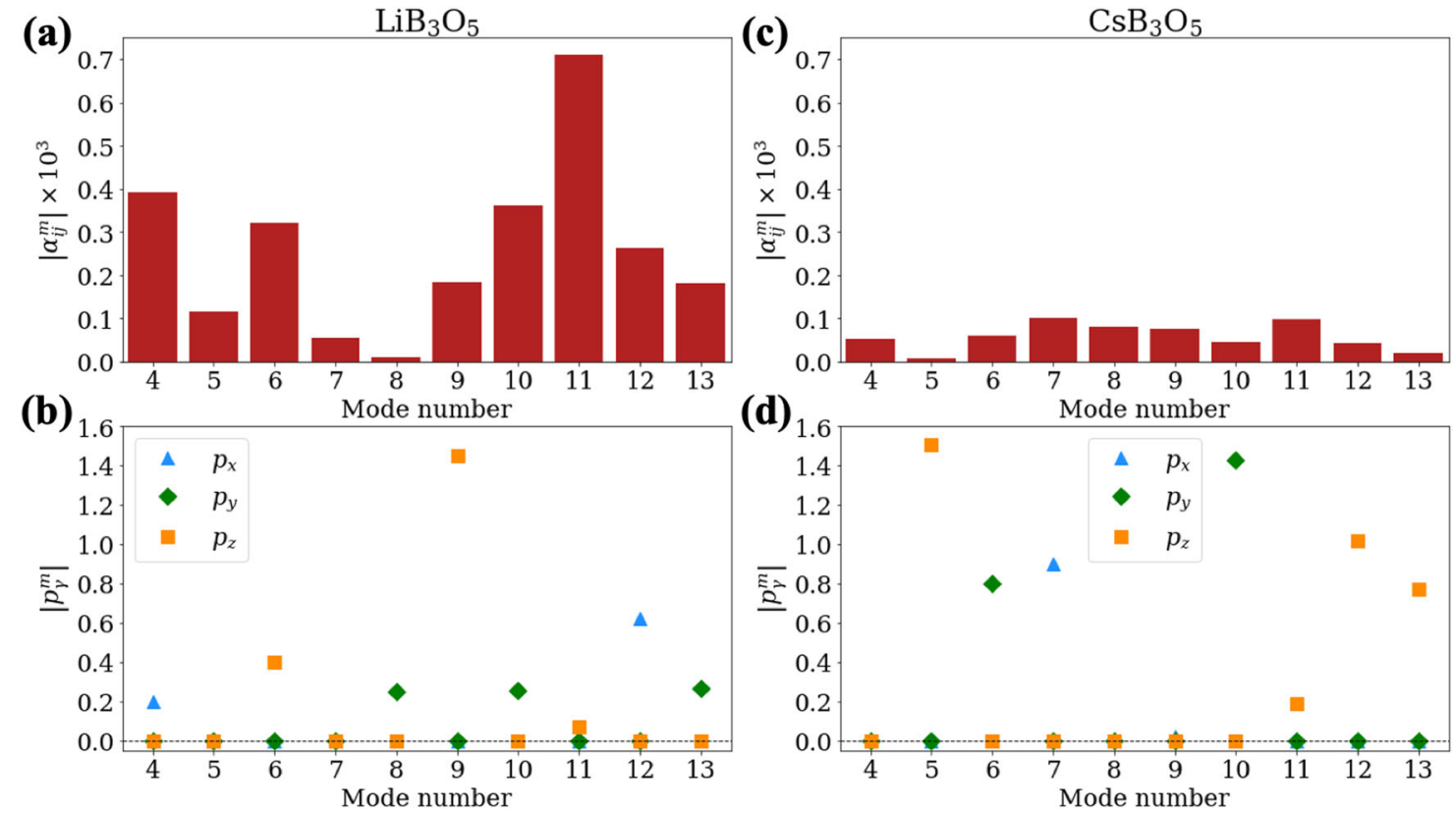

Fig. 2 Raman susceptibilities and mode polarities of LBO and CBO. For the ten softest optical modes of LBO, a the magnitude of the largest component of the Raman susceptibility, and $\mathbf{b}$ the magnitude of the mode polarity. The same quantities are plotted for CBO in $\mathbf{c}$ and $\mathbf{d}$, respectively. The Raman susceptibilities of $L B O(\mathbf{a})$ are much larger than those of $C B O(\mathbf{c})$, while the mode polarities (b, d) are roughly the same (because the atoms have Born effective charges of roughly the same magnitude). The Raman susceptibilities of LBO (a) are comparable in magnitude to those of STO at $1 \%$ biaxial compressive strain (Fig. 3a), while the mode polarities are much smaller and the phonon frequencies are much larger ${ }^{18}$

X-ray powder diffraction study ${ }^{41}$ of the thermal expansion of LBO found the driving mechanisms behind the thermal expansion in LBO to be large oscillations of the Li atom along the $a$-axis, the increasing separation of the $\mathrm{B}_{3} \mathrm{O}_{7}$ chains comprising the $c$-oriented helices (leading to the large thermal expansion in the $a$ - and $b$ directions), and the collapse of a $\mathrm{B}-\mathrm{O}-\mathrm{B}$ angle linking different chains (leading to the compression in the c-direction). Furthermore, the frequency of oscillation is proportional to $1 / \sqrt{m}$. The heaviest atom in LBO is oxygen, which is tightly bonded to the B atoms. In contrast, in perovskite titanates ${ }^{18-20}$, the most anharmonic mode (and the mode responsible for the large Pockels responses) is associated with a ferroelectric phase transition and involves displacement of a much heavier $\mathrm{Ti}$ atom. The mechanism underlying the anharmonicity of perovskite titanates is therefore quite different from LBO. Therefore, when the phonon mode driving the thermal expansion is a high-frequency mode, the simple association between strong Pockels response and anharmonicity breaks down.

Given these differences between LBO and the perovskite titanates, can the LBO crystal's Pockels response be enhanced? We calculate the Grüneisen parameters of the ten softest optical modes of LBO and find mode 9 is the most anharmonic (Fig. S1). This phonon mode primarily consists of motion of the Li atoms (Fig. S2). Owing to its relatively large Raman susceptibility and mode polarity (Fig. 2), it has a relatively high contribution to $r^{\text {ion }}$ (specifically, $r_{23}^{\text {mode } 9}=0.32 \mathrm{pm} / \mathrm{V}$ and $r_{33}^{\text {mode } 9}=-0.19 \mathrm{pm} / \mathrm{V}$ ). Only the high frequency $\left(\omega_{0}=154.9 \mathrm{~cm}^{-1}\right)$ of the mode keeps the contribution of this mode to the Pockels tensor small. If we can soften this mode, we would expect the already (relatively) large contribution of this mode to increase. 


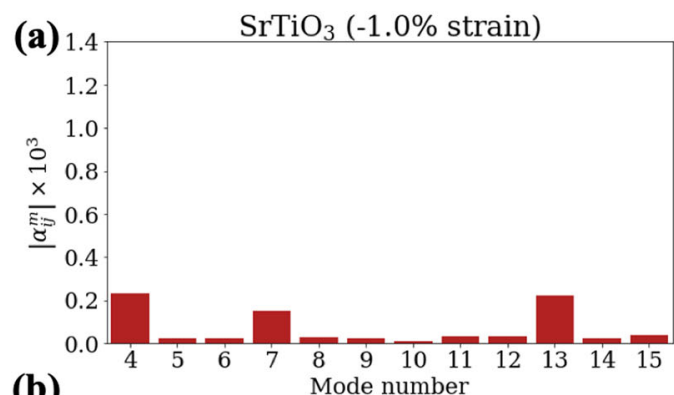

(b)

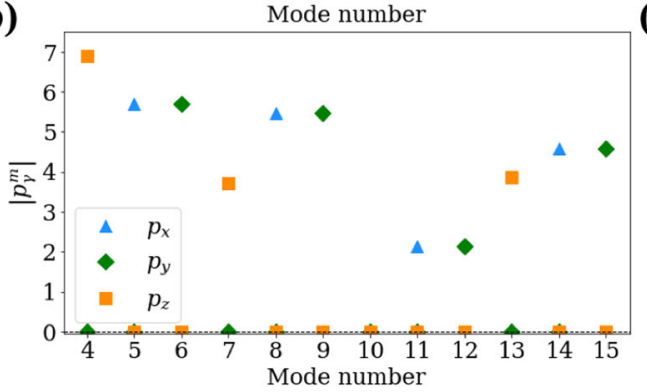

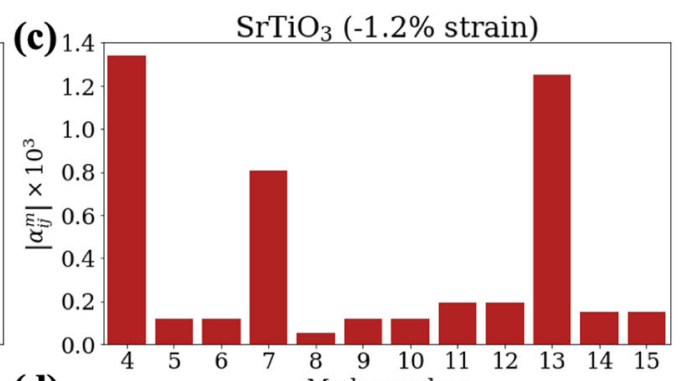

(d)

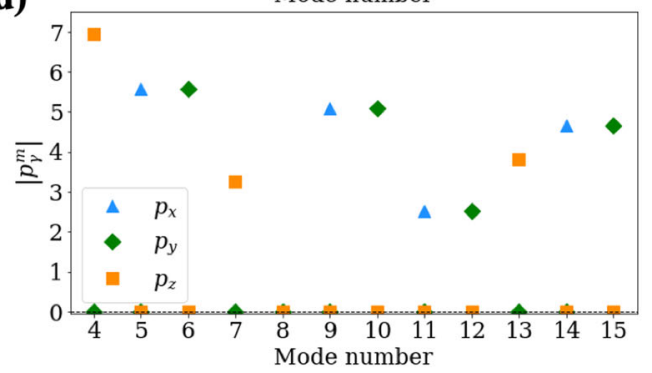

Fig. 3 Raman susceptibilities and mode polarities of strained STO. Raman susceptibilities and mode polarities for all 12 optical modes of STO (see Hamze and Demkov ${ }^{18}$ for calculation details). The magnitude of the largest component of the Raman susceptibility and the magnitude of the mode polarity under biaxial compressive strain of $1.0 \%$ are shown in $\mathbf{a}$ and $\mathbf{b}$, respectively. The same values are plotted for STO at the critical strain in $\mathbf{c}$ and $\mathbf{d}$, respectively. The magnitudes of the mode polarity are essentially unchanged under the different strains $(\mathbf{b}, \mathbf{d})$, but the Raman susceptibility increases by an order of magnitude at every mode $(\mathbf{a}, \mathbf{c})$. The frequency of the softest mode, the primary contributor to the Pockels response in STO, is also approximately halved from $-1.0 \%$ strain to $-1.2 \%$ strain.

To soften the mode, we replace the Li atoms in the crystal with $\mathrm{Cs}$ atoms. Cs is $\sim 19$ times heavier than $\mathrm{Li}$, and because the ionic contribution to the Pockels tensor is inversely proportional to the phonon frequency squared, which is in turn inversely proportional to the atomic mass, we expect a significant reduction in the phonon frequencies from this replacement. However, a simple replacement of Li with Cs in LBO leads to a dynamically unstable crystal, so we instead consider the related $\mathrm{CBO}$ crystal. Its space group is $\mathrm{P} 2{ }_{1} 2_{1} 2_{1}$, and like $\mathrm{LBO}, \mathrm{CBO}$ has a network of $\mathrm{B}_{3} \mathrm{O}_{7}$ rings, but in $\mathrm{CBO}$ they share four of their external oxygen atoms with other $\mathrm{B}_{3} \mathrm{O}_{7}$ rings ${ }^{57,58}$. The $\mathrm{Cs}$ atoms sit in the voids of this network, which run along the $a$ - and $b$-axes instead of just along one axis as in LBO. The phonon frequencies in CBO (Table S1) are indeed much lower than in LBO, but the Pockels tensor (Table 2) remains extremely small. To understand why, we now turn to the numerator of the ionic contribution to the Pockels tensor (Eq. (4)) that is the product of the Raman susceptibility (Eq. (6)) and the mode polarity (Eq. (7)). In Fig. 2, we plot the magnitude of the Raman susceptibilities and mode polarities of the ten softest optical modes of LBO and CBO. The mode polarities of LBO and $\mathrm{CBO}$ (Fig. $2 \mathrm{~b}, \mathrm{~d}$ ) are roughly of the same magnitude, which we expect because the atoms in LBO and CBO have similar Born effective charges. The Raman susceptibilities (Fig. 2a, c), however, are an order of magnitude smaller in CBO. This cancels out all the gains made from reducing the phonon frequencies and keeps the net EO response small.

To understand the role of the Raman susceptibility in the Pockels response, we first consider its role in the extreme enhancement of the Pockels response in strained $\mathrm{STO}^{18}$, and second, to gain physical intuition of the underlying mechanism, we introduce a simple tight-binding model. In Fig. 3, we plot the Raman susceptibility and mode polarity of STO under two biaxial compressive strains, $-1.0 \%$ in $\mathrm{a}$ and $\mathrm{b}$ and $-1.2 \%$ in $\mathrm{c}$ and $\mathrm{d}$. Hamze and Demkov ${ }^{18}$ showed that at $-1.2 \%$ strain (hereafter referred to as the critical strain), the Pockels tensor exhibits a large peak with the largest ionic component reaching $r_{33}=$ $-236.469 \mathrm{pm} / \mathrm{V}$. This is caused by the proximity of the crystal at this strain to a phase transition from the centrosymmetric tetragonal $P 4 / \mathrm{mmm}$ phase to the noncentrosymmetric tetragonal
P4mm phase. The phonon mode responsible for this transition goes extremely soft at this strain, leading to the large Pockels response. However, there is an additional contribution to the large response. Comparing the Raman susceptibilities at $-1.0 \%$ strain and $-1.2 \%$ strain (Fig. 3a, c, respectively), we see that the magnitude of the Raman susceptibility increases by an order of magnitude at the critical strain. In contrast, the mode polarities (Fig. 3b, d) are effectively unchanged between strains because, like in the LBO and CBO case, the Born effective charges are identical between the cells.

\section{DISCUSSION}

To gain further insight, we calculate the derivative of the electronic susceptibility with respect to atomic displacements, $\partial \chi^{(1)} / \partial \tau$, which is the key component of the Raman susceptibility (see Eq. (6)), in a simple tight-binding model ${ }^{59,60}$ of a 1-D diatomic chain with lattice constant $a$ and distance between the atoms of $a / 2$. One atom has a $1 s$ orbital and the other has a $2 p$ orbital. We assume the hopping interaction is proportional to $1 / d^{2}$ with constant of proportionality $A$, where $d$ is the distance between the atoms, which is a good approximation for many materials ${ }^{61}$. The linear electronic susceptibility in an insulating crystal is given by ${ }^{61}$

$x^{(1)}=\frac{2 \hbar^{4} e^{2}}{m_{e} \Omega} \sum_{n, n^{\prime}} \frac{\left|\left\langle\psi_{n^{\prime}}|\partial / \partial x| \psi_{n}\right\rangle\right|^{2}}{\left(\varepsilon_{n^{\prime}}-\varepsilon_{n}\right)^{3}}$,

where $n$ and $n^{\prime}$ are band indices for occupied and unoccupied bands, respectively, $\psi_{n}$ is the wave function with eigenvalue $\varepsilon_{n}$, and the band sum implicitly contains a sum over the entire Brillouin zone. Note that the susceptibility goes as the inverse cube of the transition energy, a detail that will be important later. Considering only nearest neighbor interactions, we calculate a numerical derivative of the susceptibility as a function of atomic displacement in the optical mode (see Eq. (12) and the "Methods" section for more details). For $A=1 \mathrm{eV} \cdot \AA^{2}, a=1 \AA, \varepsilon_{s}=0 \mathrm{eV}$, $\varepsilon_{p}=3 \mathrm{eV}$, and a displacement amplitude of $\delta=0.01 a$, we obtain $X^{(1)}=97.96$ and $\partial x^{(1)} / \partial \tau=-180.95 \AA^{-1}$. In other words, a $2 \%$ 

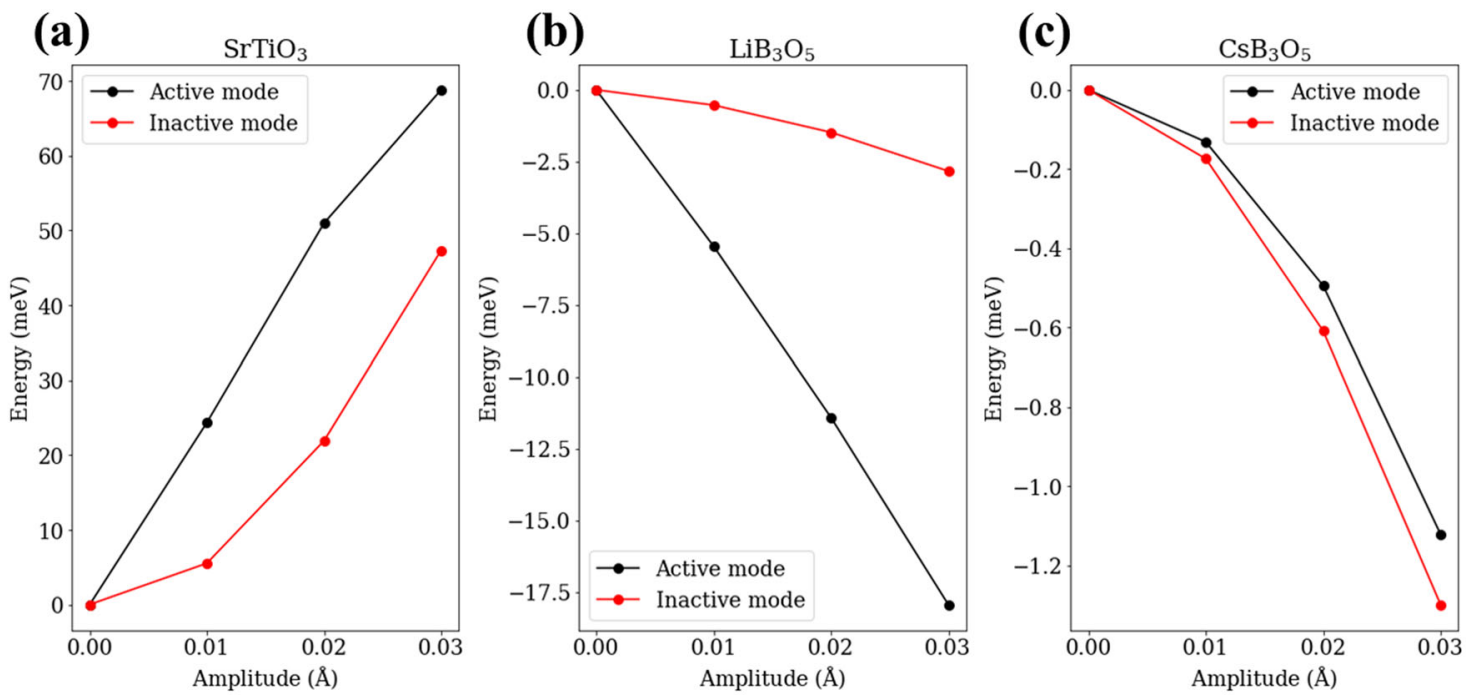

Fig. 4 Transition energies vs phonon amplitudes. Smallest transition energies as a function of amplitude of phonon mode displacements for $\mathbf{a}$ STO at the critical compressive strain ${ }^{18}, \mathbf{b} \mathrm{LBO}$, and $\mathbf{c}$ CBO. The smallest transition energy of the ground state structure for each material is set to 0 . Changes in the transition energy due to phonon modes that have large Raman susceptibilities and contribute strongly to the Pockels response (Pockels-active modes) are plotted in black, and changes in the transition energy due to phonon modes with small Raman susceptibilities and weakly contribute (or do not contribute) to the Pockels response (Pockels- inactive modes) are plotted in red.

change in the bond length amounts to a $\sim 20 \%$ change in $X^{(1)}$, showing $\partial x^{(1)} / \partial \tau$ is large.

The connection between the change in the Raman susceptibility, the electronic structure, and phonons can be understood as follows. Because of the third power in the denominator of Eq. (11), the smallest electronic transition energy is the dominant contribution to the susceptibility; hence, phonon modes that strongly affect the band edges should have large Raman susceptibilities. If those modes are soft, that in turn should ensure a large contribution to the Pockels tensor. In Fig. 4, we plot the smallest transition energy as a function the amplitude of two different phonon modes for STO, LBO, and CBO (Fig. 4a-C, respectively). The two phonon modes for each material are chosen such that one (labeled the "active" mode) has a large Raman susceptibility and a significant contribution to the Pockels tensor, while the other (labeled the "inactive" mode) does not. It is clear that in STO the active phonon causes a large change in the direct band gap, while in CBO the active mode has nearly no effect. This is because the band gap in STO, a charge transfer oxide, is controlled by the $p-d$ bonding-antibonding separation, and the active phonon changes the $\mathrm{Ti}-\mathrm{O}$ interatomic distance. This makes the differences between these three materials clear: STO has soft phonons and the soft modes strongly affect the band edges, which leads to a large Raman susceptibility and EO response. The active mode in LBO also has a significant effect on the gap and therefore the Raman susceptibility is relatively large, but the high phonon frequencies suppress the EO response. In $C B O$, this is reversed with very soft modes having very little impact on the band gap, which therefore suppresses the Raman susceptibility and the EO response.

This aspect of electron-phonon interactions is commonly described using deformation potentials ${ }^{54-56}$, which we list for STO, LBO, and CBO in Table S2 (we also provide the band structure of $\mathrm{LBO}$ and $\mathrm{CBO}$ in Fig. S3). We see that the deformation potential for every material is larger when the ions are displaced according to the Pockels active mode than when they are displaced according to the inactive mode (except in $\mathrm{CBO}$, in which the deformation potentials for both modes are $\sim 0 \mathrm{eV} / \AA ̊$ ), indicating that large deformation potentials are associated with large Raman susceptibilities and therefore could indicate the potential for strong Pockels responses in such materials.
To summarize, soft phonons inducing substantial changes in the band gap (in other words those with strong electron-phonon couplings) cause large changes in the electronic susceptibility, as the tight-binding model above illustrates. Large changes in the electronic susceptibility in turn imply large Raman susceptibilities, to which the ionic contribution to the EO response is proportional. Therefore, noncentrosymmetric materials with soft phonons and strong electron-phonon interaction will likely be strong Pockels materials. This explains why LBO and CBO have weak EO responses, and why perovskite titanates are special and have such strong EO responses.

We explore the relationship between the microscopic properties such as lattice anharmonicity and strength of electron-phonon coupling and the Pockels effect using LBO as a test case because of its extraordinarily large thermal expansion coefficients. Despite the correspondence between large thermal expansion coefficients and large EO responses many EO materials, we found that LBO has a very small Pockels response. The small Pockels response is caused by the extremely small masses of the constituent atoms resulting in high optical phonon frequencies.

Guided by the Grüneisen parameter analysis of the ten softest optical phonon modes, we tested this assertion by studying the related CBO crystal to see if simply increasing the atomic masses would lead to a large Pockels response by softening the modes. This approach was found to be too simplistic -in CBO, the small Raman susceptibility results in a weak Pockels response despite the presence of much softer phonon modes. A simple tight binding model provides an intuitive physical description of the underlying mechanism at play behind the Raman susceptibility. Borrowing from the methods used to study semiconductors, this can be explained in terms of the deformation potential, which quantifies the strength of the electron-phonon coupling of the near-edge states. Modes with large Raman susceptibilities are shown to have large deformation potentials. Hence, we formulate a refined design rule for strong Pockels materials: materials with strong electron-phonon couplings (and therefore large Raman susceptibilities), that are noncentrosymmetric, and that have soft phonons will be strong Pockels materials. We thus show the most important components of the ionic Pockels response are the phonon frequency softness (for which the thermal expansion and Grüneisen parameters are proxies) and Raman susceptibility 
(strong electron-phonon coupling). Our results will hopefully guide materials scientists and engineers in searching for new EO materials with even stronger responses.

\section{METHODS}

\section{DFT calculations}

All DFT and density functional perturbation theory calculations were performed using the ABINIT software package ${ }^{45-51}$. Troullier-Martins normconserving pseudopotentials ${ }^{62}$ generated with the fhi98PP package ${ }^{63}$ were used for all calculations. The valence electron configurations were $2 s^{1}$ for $\mathrm{Li}, 2 s^{2} 2 p^{1}$ for $\mathrm{B}, 2 s^{2} 2 p^{4}$ for $\mathrm{O}$, and $6 s^{1}$ for Cs. The exchange-correlation energy was calculated in the local density approximation ${ }^{64,65}$. An $8 \times 8 \times 8$ Monkhorst-Pack ${ }^{66,67} k$-point grid and a plane wave cut-off energy of $50 \mathrm{Ha}$ was used for all calculations. The lattice parameters used for all the LBO calculations are $a=8.444 \AA=15.957 \mathrm{Bohr}, b=7.378 \AA=13.942 \mathrm{Bohr}$, and $c=5.146 \AA=9.725$ Bohr, which are experimental lattice parameters measured at room temperature by Shepelev et al. ${ }^{41}$. The atomic positions are fully relaxed from the initial experimental coordinates until the forces were smaller than $2 \times 10^{-5} \mathrm{Ha} /$ Bohr.

For calculations of $\mathrm{CBO}$, we use the experimental structure determined by Krogh-Moe ${ }^{57}$, which has lattice parameters $a=6.213 \AA=11.741$ Bohr $b=8.521 \AA=16.102 \mathrm{Bohr}$, and $c=9.170 \AA=17.329$ Bohr.

To calculate the piezoelectric contribution to the Pockels tensor, we need to calculate the photoelastic tensor (see Eq. (4)). We do this by straining the unit cell to isolate the component of the tensor we want, calculating the dielectric tensor, inverting it, and taking a numerical derivative with respect to strain $^{18,53,68}$.

In Eq. (11), we calculate the matrix element in the numerator following Graf and Vogl ${ }^{69}$. For a given $k^{*}$, it can be calculated as ${ }^{69}$

$\left\langle\psi_{n^{\prime}}|\partial / \partial x| \psi_{n}\right\rangle=\frac{m_{e}}{\hbar} \sum_{\alpha^{\prime}, a} C_{\alpha^{\prime}}^{*}\left(n k^{*}\right) \sum_{L} i\left(R_{\alpha^{\prime}, L}-R_{a, 0}\right) e^{i k^{*}\left(R_{\alpha^{\prime}, L}-R_{a, 0}\right)} t_{\alpha^{\prime}, a}\left(R_{\alpha^{\prime}, L}-R_{a, 0}\right) C_{a}\left(n^{\prime} k^{*}\right)$,

where $a$ is a compound index that labels the atomic orbital and the position of the atom in the cell, $L$ (and 0) label the unit cell, $n$ is a band index, the $C_{a}$ are the coefficients in the expansion of the eigenfunctions of the tight-binding Hamiltonian, and $t_{\alpha^{\prime}, a}\left(R_{\alpha^{\prime}, L}-R_{a, 0}\right)=\left\langle\alpha^{\prime}, L|H| a, 0\right\rangle$.

\section{DATA AVAILABILITY}

Requests for data should be addressed to A.A.D., demkov@physics.utexas.edu.

Received: 29 February 2020; Accepted: 28 July 2020;

Published online: 21 August 2020

\section{REFERENCES}

1. Wessels, B. W. Ferroelectric epitaxial thin films for integrated optics. Annu. Rev. Mater. Res. 37, 659-679 (2007)

2. Reed, G. T., Mashanovich, G., Gardes, F. Y. \& Thomson, D. J. Silicon optical modulators. Nat. Photonics 4, 518-526 (2010).

3. Heck, M. J. R. et al. Hybrid silicon photonics for optical interconnects. IEEE J. Sel. Top. Quantum Electron 17, 333-346 (2011).

4. Gourlay, J., Forbes, M. \& Desmulliez, M. Optically interconnected electronic chips: a tutorial and review of the technology. Electron. Commun. Eng. J. 13, 221-232 (2001).

5. Benner, A. F., Ignatowski, M., Kash, J. A., Kuchta, D. M. \& Ritter, M. B. Exploitation of optical interconnects in future server architectures. IBM J. Res. Dev. 49, 755-775 (2005)

6. Li, M. \& Tang, H. X. Strong Pockels materials. Nat. Mater. 18, 9-11 (2019).

7. Abel, S. et al. Large Pockels effect in micro- and nanostructured barium titanate integrated on silicon. Nat. Mater. 18, 42-47 (2019).

8. Ladd, T. D. et al. Quantum computers. Nature 464, 45-53 (2010).

9. O’Brien, J. L., Furusawa, A. \& Vučković, J. Photonic quantum technologies. Nat. Photonics 3, 687-695 (2009).

10. Boyd, R. Nonlinear Optics (Academic Press, 2008).

11. Jazbinšek, M. \& Zgonik, M. Material tensor parameters of LiNbO3 relevant for electro- and elasto-optics. Appl. Phys. B Lasers Opt. 74, 407-414 (2002).

12. Wooten, E. L. et al. A review of lithium niobate modulators for fiber-optic communications systems. IEEE J. Sel. Top. Quantum Electron. 6, 69-82 (2000),
13. Janner, D., Tulli, D., Garcia-Granda, M., Belmonte, M. \& Pruneri, V. Microstructured integrated electro-optic $\mathrm{LiNbO}_{3}$ modulators. Laser Photonics Rev. 3, 301-313 (2009).

14. Chen, L. \& Reano, R. M. Compact electric field sensors based on indirect bonding of lithium niobate to silicon microrings. Opt. Express 20, 4032-4038 (2012).

15. Chen, L., Xu, Q., Wood, M. G. \& Reano, R. M. Hybrid silicon and lithium niobate electro-optical ring modulator. Optica 1, 112-118 (2014).

16. Wang, C., Zhang, M., Stern, B., Lipson, M. \& Lončar, M. Nanophotonic lithium niobate electro-optic modulators. Opt. Express 26, 1547-1555 (2018).

17. Eltes, F. et al. A novel 25 Gbps electro-optic Pockels modulator integrated on an advanced Si photonic platform. In 2017 IEEE International Electron Devices Meeting (IEDM) 24.5.1-24.5.4 (IEEE, 2017). https://doi.org/10.1109/ IEDM.2017.8268454.

18. Hamze, A. K. \& Demkov, A. A. First-principles study of the linear electro-optical response in strained $\mathrm{SrTiO}_{3}$. Phys. Rev. Mater. 2, 115202 (2018).

19. Fredrickson, K. D. et al. Strain enhancement of the electro-optical response in $\mathrm{BaTiO}_{3}$ films integrated on Si(001). Phys. Rev. B 98, 075136 (2018).

20. Paillard, C., Prokhorenko, S. \& Bellaiche, L. Strain engineering of electro-optic constants in ferroelectric materials. npj Comput. Mater. 5, 6 (2019).

21. Veithen, M., Gonze, X. \& Ghosez, P. First-principles study of the electro-optic effect in ferroelectric oxides. Phys. Rev. Lett. 93, 187401 (2004).

22. Castera, P. et al. Electro-optical modulation based on Pockels effect in $\mathrm{BaTiO}_{3}$ with a multi-domain structure. IEEE Photonics Technol. Lett. 28, 990-993 (2016).

23. Abel, S. et al. A strong electro-optically active lead-free ferroelectric integrated on silicon. Nat. Commun. 4, 1671 (2013).

24. Abel, S. et al. A hybrid barium titanate-silicon photonics platform for ultraefficient electro-optic tuning. J. Light. Technol. 34, 1688-1693 (2016).

25. Kormondy, K. J. et al. Microstructure and ferroelectricity of $\mathrm{BaTiO}_{3}$ thin films on Si for integrated photonics. Nanotechnology 28, 075706 (2017).

26. Eltes, $\mathrm{F}$. et al. Low-loss $\mathrm{BaTiO}_{3}-\mathrm{Si}$ waveguides for nonlinear integrated photonics. ACS Photonics 3, 1698-1703 (2016).

27. Kormondy, K. J. et al. Analysis of the Pockels effect in ferroelectric barium titanate thin films on Si(001). Microelectron. Eng. 147, 215-218 (2015).

28. Dubourdieu, C. et al. Switching of ferroelectric polarization in epitaxial $\mathrm{BaTiO}_{3}$ films on silicon without a conducting bottom electrode. Nat. Nanotechnol. 8, 748-754 (2013).

29. Ma, H., Levy, J., Biegalski, M. D., Trolier-McKinstry, S. \& Schlom, D. G. Roomtemperature electro-optic properties of strained $\mathrm{SrTiO}_{3}$ films grown on $\mathrm{DySCO}_{3}$. J. Appl. Phys. 105, 014102 (2009).

30. Rosa, A. et al. Barium titanate $\left(\mathrm{BaTiO}_{3}\right)$ RF characterization for application in electro-optic modulators. Opt. Mater. Express 7, 4328-4336 (2017).

31. Lin, E. L. et al. Atomic layer deposition of epitaxial ferroelectric barium titanate on Si(001) for electronic and photonic applications. J. Appl. Phys. 126, 064101 (2019).

32. Xiong, C. et al. Active silicon integrated nanophotonics: ferroelectric $\mathrm{BaTiO}_{3}$ devices. Nano Lett. 14, 1419-1425 (2014).

33. McKee, R. A., Walker, F. J., Conner, J. R., Specht, E. D. \& Zelmon, D. E. Molecular beam epitaxy growth of epitaxial barium silicide, barium oxide, and barium titanate on silicon. Appl. Phys. Lett. 59, 782-784 (1991).

34. Zgonik, M. et al. Dielectric, elastic, piezoelectric, electro-optic, and elasto-optic tensors of $\mathrm{BaTiO}_{3}$ crystals. Phys. Rev. B 50, 5941-5949 (1994).

35. Beattie, A. G. \& Samara, G. A. Pressure dependence of the elastic constants of $\mathrm{SrTiO}_{3}$. J. Appl. Phys. 42, 2376-2381 (1971).

36. Brugger, K. Generalized Grüneisen parameters in the anisotropic debye model Phys. Rev. 137, A1826-A1827 (1965).

37. Kurtz, S. K. \& Robinson, F. N. H. A physical model of the electro-optic effect. Appl. Phys. Lett. 10, 62-65 (1967).

38. Nikogosyan, D. N. Lithium triborate (LBO). Appl. Phys. A Solids Surf. 58, 181-190 (1994).

39. Chen, C. et al. New nonlinear-optical crystal: $\mathrm{LiB3O}_{5}$. J. Opt. Soc. Am. B 6, 616-621 (1989).

40. Lin, S., Sun, Z., Wu, B. \& Chen, C. The nonlinear optical characteristics of a $\mathrm{LiB}_{3} \mathrm{O}_{5}$ crystal. J. Appl. Phys. 67, 634-638 (1990).

41. Shepelev, Y. F., Bubnova, R. S., Filatov, S. K., Sennova, N. A. \& Pilneva, N. A. LiB $\mathrm{O}_{5}$ crystal structure at 20, 227 and $377^{\circ} \mathrm{C}$. J. Solid State Chem. 178, 2987-2997 (2005).

42. Wei, L., Guiqing, D., Qingzhen, H., An, Z. \& Jingkui, L. Anisotropic thermal expansion of $\mathrm{LiB}_{3} \mathrm{O}_{5}$. J. Phys. D. Appl. Phys. 23, 1073-1075 (1990).

43. Johnston, W. D. \& Kaminow, I. P. Contributions to optical nonlinearity in GaAs as determined from raman scattering efficiencies. Phys. Rev. 188, 1209-1211 (1969).

44. Johnston, W. D. Nonlinear optical coefficients and the raman scattering efficiency of LO and TO phonons in acentric insulating crystals. Phys. Rev. B 1, 3494-3503 (1970). 
45. Gonze, X. A brief introduction to the ABINIT software package. Z. f.ür. Krist. Cryst. Mater. 220, 558-562 (2005).

46. Gonze, X. et al. ABINIT: first-principles approach to material and nanosystem properties. Comput. Phys. Commun. 180, 2582-2615 (2009).

47. Gonze, X. et al. Recent developments in the ABINIT software package. Comput. Phys. Commun. 205, 106-131 (2016).

48. Hamann, D. R., Wu, X., Rabe, K. M. \& Vanderbilt, D. Metric tensor formulation of strain in density-functional perturbation theory. Phys. Rev. B 71, 035117 (2005).

49. Veithen, M., Gonze, X. \& Ghosez, P. Nonlinear optical susceptibilities, Raman efficiencies, and electro-optic tensors from first-principles density functional perturbation theory. Phys. Rev. B 71, 125107 (2005).

50. Gonze, X. First-principles responses of solids to atomic displacements and homogeneous electric fields: implementation of a conjugate-gradient algorithm. Phys. Rev. B 55, 10337-10354 (1997).

51. Gonze, X. \& Lee, C. Dynamical matrices, Born effective charges, dielectric permittivity tensors, and interatomic force constants from density-functional perturbation theory. Phys. Rev. B 55, 10355-10368 (1997).

52. Fontana, M. D., Laabidi, K., Jannot, B., Maglione, M. \& Jullien, P. Relationship between electro-optic, vibrational and dielectric properties in $\mathrm{BaTiO}_{3}$. Solid State Commun. 92, 827-830 (1994).

53. Nye, J. F. Physical Properties of Crystals (Oxford University Press, 1985).

54. Bardeen, J. \& Shockley, W. Deformation potentials and mobilities in non-polar crystals. Phys. Rev. 80, 72-80 (1950).

55. Pötz, W. \& Vogl, P. Theory of optical-phonon deformation potentials in tetrahedral semiconductors. Phys. Rev. B 24, 2025-2037 (1981).

56. Blacha, A., Presting, H. \& Cardona, M. Deformation potentials of $k=0$ states of tetrahedral semiconductors. Phys. Status Solidi 126, 11-36 (1984).

57. Krogh-Moe, J. Refinement of the crystal structure of caesium triborate, $\mathrm{Cs}_{2} \mathrm{O} .3 \mathrm{~B}_{2} \mathrm{O}_{3}$. Acta Crystallogr. Sect. B 30, 1178-1180 (1974).

58. $\mathrm{Wu}$, Y. et al. $\mathrm{CsB}_{3} \mathrm{O}_{5}$ : a new nonlinear optical crystal. Appl. Phys. Lett. 62, 2614-2615 (1993).

59. Slater, J. C. \& Koster, G. F. Simplified LCAO Method for the periodic potential problem. Phys. Rev. 94, 1498-1524 (1954).

60. Ashcroft, N. W. \& Mermin, N. D. Solid State Physics (Brooks/Cole, 1976).

61. Harrison, W. A. Electronic Structure and the Properties of Solids: The Physics of the Chemical Bond (Dover Publications, 1989).

62. Troullier, N. \& Martins, J. L. Efficient pseudopotentials for plane-wave calculations. Phys. Rev. B 43, 8861-8869 (1991).

63. Fuchs, M. \& Scheffler, M. Ab initio pseudopotentials for electronic structure calculations of poly-atomic systems using density-functional theory. Comput. Phys. Commun. 119, 67-98 (1999).

64. Ceperley, D. M. \& Alder, B. J. Ground state of the electron gas by a stochastic method. Phys. Rev. Lett. 45, 566-569 (1980).

65. Perdew, J. P. \& Zunger, A. Self-interaction correction to density-functional approximations for many-electron systems. Phys. Rev. B 23, 5048-5079 (1981).

66. Monkhorst, H. J. \& Pack, J. D. Special points for Brillouin-zone integrations. Phys. Rev. B 13, 5188-5192 (1976).

67. Pack, J. D. \& Monkhorst, H. J. 'Special points for Brillouin-zone integrations'-a reply. Phys. Rev. B 16, 1748-1749 (1977).

68. Zwanziger, J. W. et al. Finite homogeneous electric fields in the projector augmented wave formalism: applications to linear and nonlinear response. Comput. Mater. Sci. 58, 113-118 (2012).

69. Graf, M. \& Vogl, P. Electromagnetic fields and dielectric response in empirical tight-binding theory. Phys. Rev. B 51, 4940-4949 (1995).

70. $\mathrm{Xu}, \mathrm{H}$. et al. Growth and characterization of $\mathrm{Nd}^{\mathrm{Bi}} \mathrm{Bi}_{12} \mathrm{SiO}_{20}$ single crystal. Opt. Commun. 285, 3961-3966 (2012).

71. Oberschmid, R. Absorption centers of $\mathrm{Bi}_{12} \mathrm{GeO}_{20}$ and $\mathrm{Bi}_{12} \mathrm{SiO}_{20}$ crystals. Phys. Status Solidi 89, 263-270 (1985).

72. Yariv, A. \& Yeh, P. Optical Waves in Crystals: Propagation and Control of Laser Radiation (Wiley, 1984).

73. Vedam, K. \& Hennessey, P. Piezo- and thermo-optical properties of $\mathrm{Bi}_{12} \mathrm{GeO}_{20}$, II. Refractive index. J. Opt. Soc. Am. 65, 442 (1975).

74. Papazoglou, D. G., Apostolidis, A. G. \& Vanidhis, E. D. Index of refraction, optical activity and electro-optic coefficient of bismuth titanium oxide $\left(\mathrm{Bi}_{12} \mathrm{TiO}_{20}\right)$. Appl. Phys. B 65, 499-503 (1997).

75. Yao, W. F. et al. Photocatalytic property of bismuth titanate $\mathrm{Bi}_{12} \mathrm{TiO}_{20}$. Crystals 243, 185-190 (2011).

76. Mamoun, S., Merad, A. E. \& Guilbert, L. Energy band gap and optical properties of lithium niobate from ab initio calculations. Comput. Mater. Sci. 79, 125-131 (2013).

77. Weis, R. S. \& Gaylord, T. K. Lithium niobate: summary of physical properties and crystal structure. Appl. Phys. A 37, 191-203 (1985).
78. Kim, Y. S. \& Smith, R. T. Thermal expansion of lithium tantalate and lithium niobate single crystals. J. Appl. Phys. 40, 4637-4641 (1969).

79. Casson, J. L. et al. Electro-optic coefficients of lithium tantalate at near-infrared wavelengths. J. Opt. Soc. Am. B 21, 1948-1952 (2010).

80. Ismangil, A., Jenie, R. P., Irmansyah, E. \& Irzaman, H. Development of lithium tantallite $\left(\mathrm{LiTaO}_{3}\right)$ for automatic switch on LAPAN-IPB satellite infra-red sensor. Procedia Environ. Sci. 24, 329-334 (2015).

81. Abel, S. Dispositifs électro-optiques à base de Titanate de Baryum épitaxié sur Silicium pour la photonique intégrée. (Université de Grenoble, 2014).

82. Wemple, S. H., Didomenico, M. \& Camlibel, I. Dielectric and optical properties of melt- grown $\mathrm{BaTiO}_{3}$. J. Phys. Chem. Solids 29, 1797-1803 (1968).

83. Suzuki, K. \& Kijima, K. Optical band gap of barium titanate nanoparticles prepared by RF-plasma chemical vapor deposition. Jpn. J. Appl. Phys. 44, 2081-2082 (2005).

84. Paszkowski, R., Wokulska, K. \& Dec, J. Thermal expansion coefficients of strontium-barium niobate single crystals in the vicinity of the phase transition point. Cryst. Res. Technol. 52, 4-7 (2017).

85. Dorywalski, K. et al. Ellipsometric study of near band gap optical properties of SrxBa1- $\mathrm{xNb}_{2} \mathrm{O}_{6}$ crystals. Opt. Mater. 35, 887-892 (2013).

86. Podlozhenov, $\mathrm{S}$. et al. Structure of strontium barium niobate $\mathrm{Sr}_{x} \mathrm{Ba}_{1-x} \mathrm{Nb}_{2} \mathrm{O}_{6}$ (SBN) in the composition range $0.32 \leq \mathrm{x} \leq 0.82$. Acta Crystallogr. Sect. B 62 , 960-965 (2006).

87. Photorefractive Crystals SBN, BSO, BGO, Fe:LNB. 1-2. http://www.mt-berlin.com/ frames_cryst/descriptions/photoref.htm\# (2016).

88. Trivedi, D., Tayebati, P. \& Tabat, M. Measurement of large electro-optic coefficients in thin films of strontium barium niobate $\left(\mathrm{Sr}_{0.6} \mathrm{Ba}_{0.4} \mathrm{Nb}_{2} \mathrm{O}_{6}\right)$. Appl. Phys. Lett. 68, 3227-3229 (1996).

89. Jamieson, P. B. Ferroelectric tungsten bronze-type crystal structures. I. Barium strontium niobate $\mathrm{Ba}_{0.27} \mathrm{Sr}_{0.75} \mathrm{Nb}_{2} \mathrm{O}_{5.78}$. J. Chem. Phys. 48, 5048-5057 (1968).

90. Qadri, S. B. et al. Phase transition in $\mathrm{Sr}_{0.75} \mathrm{Ba}_{0.25} \mathrm{NbO}_{3}$ near the Curie temperature. Appl. Phys. Lett. 89, 222911 (2006).

91. Tayebati, P., Trivedi, D. \& Tabat, M. Pulsed laser deposition of SBN:75 thin films with electro-optic coefficient of $844 \mathrm{pm} / \mathrm{N}$. Appl. Phys. Lett. 69, 1023-1025 (1996).

92. Ghosh, G. Dispersion of thermo-optic coefficients in a potassium niobate nonlinear crystal. Appl. Phys. Lett. 65, 3311-3313 (1994).

93. Wiesendanger, E. Dielectric, mechanical and optical properties of orthorhombic $\mathrm{KNbO}_{3}$. Ferroelectrics 6, 263-281 (1973).

94. Zgonik, M., Schlesser, R., Biaggio, I. \& Günter, P. Electro- and acousto-optic properties of $\mathrm{KNbO}_{3}$ crystals. Ferroelectrics 158, 217-222 (1994).

95. Jiangou, Z., Shipin, Z., Dingquan, X., Xiu, W. \& Guanfeng, X. Optical absorption properties of doped lithium niobate crystals. J. Phys. Condens. Matter 4, 2977-2983 (1992).

96. Optical Grade Lithium Niobate, Lithium Tantalate. http://www.roditi.com/ SingleCrystal/LiNbO3/Magnesium-Doped.html (2004).

97. French, R. H., Ling, J. W., Ohuchi, F. S. \& Chen, C. T. Electronic structure of $\beta-\mathrm{BaB} 2 \mathrm{O} 4$ and LiB3O5 nonlinear optical crystals. Phys. Rev. B 44, 8496-8502 (1991).

98. Bubnova, R., Volkov, S., Albert, B. \& Filatov, S. Borates-crystal structures of prospective nonlinear optical materials: high anisotropy of the thermal expansion caused by anharmonic atomic vibrations. Crystals 7, 93 (2017).

99. Ebbers, C. A. Linear electro-optic effect in $\beta$-BaB2O4. Appl. Phys. Lett. 52, 1948-1949 (1988).

100. Pignatiello, F. et al. Measurement of thermal expansion and thermo-optic coefficients in $\mathrm{LiNbO}_{3}$ and $\mathrm{KTTOPO}_{4}$ crystals using dual-interferometric techniques. In Proc. SPIE 6341, Speckle06: Speckles, From Grains to Flowers (eds Slangen, P. \& Cerruti, C.) $63412 \mathrm{H}$ (2006). https://doi.org/10.1117/12.695504.

101. Yang, F. et al. An energy band engineering design to enlarge the band gap of $\mathrm{KTiOPO}_{4}$ (KTP)-type sulfates via aliovalent substitution. J. Mater. Chem. C 7, 8131-8138 (2019).

102. Kato, K. \& Takaoka, E. Sellmeier and thermo-optic dispersion formulas for KTP. Appl. Opt. 41, 5040-5044 (2002).

103. Yutsis, I., Kirshner, B. \& Arie, A. Temperature-dependent dispersion relations for $\mathrm{RbTiOPO}_{4}$ and $\mathrm{RbTiOAsO}_{4}$. Appl. Phys. B 79, 77-81 (2004).

104. Tu, C.-S., Katiyar, R. S., Hugo Schmidt, V., Guo, R. \& Bhalla, A. S. Hypersonic anomalies and optical properties of $\mathrm{RbTiOAsO}_{4}$ and $\mathrm{KTiOPO}_{4}$ single crystals. Phys. Rev. B 59, 251-256 (2002).

105. Taylor, T. R. et al. Impact of thermal strain on the dielectric constant of sputtered barium strontium titanate thin films. Appl. Phys. Lett. 80, 1978-1980 (2002).

106. Bain, A. K. et al. Optical properties of barium strontium titanate (BST) ferroelectric thin films. Ferroelectr. Lett. Sect. 34, 149-154 (2007).

107. Panda, B., Dhar, A., Nigam, G. D., Bhattacharya, D. \& Ray, S. K. Optical properties of RF sputtered strontium substituted barium titanate thin films. Thin Solid Films 332, 46-49 (1998). 
108. Li, J. et al. Electro-optic measurements of the ferroelectric-paraelectric boundary in $\mathrm{Ba} 1-x \mathrm{SrxTiO}_{3}$ materials chips. Appl. Phys. Lett. 76, 769-771 (2000).

109. Kallaev, S. N. et al. Thermophysic properties of piezoelectric PZT ceramics https://thermophysics.ru/pdf doc/AutoPlay/Docs/CollectionOfManuscripts/ ECTP2005paper252.pdf.

110. Pandey, S. K. et al. Structural, ferroelectric and optical properties of PZT thin films. Phys. B Condens. Matter 369, 135-142 (2005).

111. Peng, C. H., Chang, J.-F. \& Desu, S. B. Optical properties of PZT, PLZT, and PNZT thin films. MRS Proceedings 243, 21-26 (1991).

112. Kang, H.-S. \& Lee, W.-J. Effects of deposition temperature and seed layer on the optical properties of lead zirconate titanate films. J. Vac. Sci. Technol. A 20, 1498-1504 (2002)

113. Spirin, V. V., Lee, C. \& No, K. Measurement of the Pockels coefficient of lead zirconate titanate thin films by a two-beam polarization interferometer with a reflection configuration. J. Opt. Soc. Am. B 15, 1940-1946 (1998)

114. Roessler, D. M., Walker, W. C. \& Loh, E. Electronic spectrum of crystalline beryllium oxide. J. Phys. Chem. Solids 30, 157-167 (1969).

115. Edwards, D. \& White, R. Beryllium oxide (BeO). in Handbook of Optical Constants of Solids 805-814 (Elsevier, 1997). https://doi.org/10.1016/B978-012544415-6/50081-9

116. Slack, G. A. \& Bartram, S. F. Thermal expansion of some diamondlike crystals. J. Appl. Phys. 46, 89-98 (1975).

117. Hazen, R. M. \& Finger, L. W. High-pressure and high-temperature crystal chemistry of beryllium oxide. J. Appl. Phys. 59, 3728-3733 (1986).

118. Holm, B., Ahuja, R., Yourdshahyan, Y., Johansson, B. \& Lundqvist, B. I. Elastic and optical properties of a- and $\mathrm{k}-\mathrm{Al}_{2} \mathrm{O}_{3}$. Phys. Rev. B 59, 12777-12787 (1999).

119. Halvarsson, M., Langer, V. \& Vuorinen, S. Determination of the thermal expansion

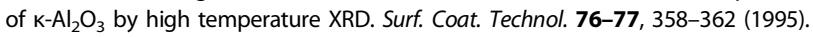

120. Batra, V., Kotru, S., Varagas, M. \& Ramana, C. V. Optical constants and band gap determination of $\mathrm{Pb}_{0.95} \mathrm{La}_{0.05} \mathrm{Zr}_{0.54} \mathrm{Ti}_{0.46} \mathrm{O}_{3}$ thin films using spectroscopic ellipsometry and UV-visible spectroscopy. Opt. Mater. 49, 123-128 (2015).

121. Okuyama, M., Usuki, T., Hamakawa, Y. \& Nakagawa, T. Epitaxial growth of ferroelectric PLZT thin film and their optical properties. Appl. Phys. 21, 339-343 (1980)

122. Thacher, P. D. Refractive index and surface layers of ceramic $(\mathrm{Pb}, \mathrm{La})(\mathrm{Zr}, \mathrm{Ti}) \mathrm{O}_{3}$ compounds. Appl. Opt. 16, 3210-3213 (2008).

123. Reitze, D. H. et al. Electro-optic properties of single crystalline ferroelectric thin films. Appl. Phys. Lett. 63, 596-598 (1993).

124. Benedek, G., Boscolo, I., Handerek, J. \& Riege, H. Electron emission from ferroelectric/antiferroelectric cathodes excited by short high-voltage pulses. J. Appl. Phys. 81, 1396-1403 (1997).

125. Madelung, O. Semiconductors: Data Handbook, Vol. 53 (Springer Berlin Heidelberg, 2004).

126. Villars, P. \& Cenzual, K. Pearson's Crystal Data: Crystal Structure Database for Inorganic Compounds. ASM International ${ }^{\circledR}$, Materials Park, Ohio, USA.

127. Ihara, M., Yuge, M. \& Krogh-Moe, J. Crystal structure of lithium triborate, $\mathrm{Li}_{2} \mathrm{O}$ $3 \mathrm{~B}_{2} \mathrm{O}_{3}$. J. Ceram. Assoc. Jpn. 88, 179-184 (1980).

128. König, H. \& Hoppe, R. Uber Borati der Alkalimetalle. II. Zur Kenntnis von $\mathrm{LiB}_{3} \mathrm{O}_{5}$. Z. für Anorg. und Allg. Chem. 439, 71-79 (1978).

\section{ACKNOWLEDGEMENTS}

The authors would like to thank Agham Posadas and Elliott Ortmann for critical reading of the manuscript. This work is supported by the Air Force Office of Scientific Research under Grant FA9550-18-1-0053. All calculations are performed at the Texas Advanced Computing Center.

\section{AUTHOR CONTRIBUTIONS}

A.K.H. did all the calculations and data analysis. M.R., J.G.-K., and A.K.H. made Table 1. All authors contributed to writing the manuscript. A.A.D. supervised and lead the study.

\section{COMPETING INTERESTS}

The authors declare no competing interests.

\section{ADDITIONAL INFORMATION}

Supplementary information is available for this paper at https://doi.org/10.1038/ s41524-020-00399-z.

Correspondence and requests for materials should be addressed to A.A.D.

Reprints and permission information is available at http://www.nature.com/ reprints

Publisher's note Springer Nature remains neutral with regard to jurisdictional claims in published maps and institutional affiliations.

Open Access This article is licensed under a Creative Commons Attribution 4.0 International License, which permits use, sharing, adaptation, distribution and reproduction in any medium or format, as long as you give appropriate credit to the original author(s) and the source, provide a link to the Creative Commons license, and indicate if changes were made. The images or other third party material in this article are included in the article's Creative Commons license, unless indicated otherwise in a credit line to the material. If material is not included in the article's Creative Commons license and your intended use is not permitted by statutory regulation or exceeds the permitted use, you will need to obtain permission directly from the copyright holder. To view a copy of this license, visit http://creativecommons. org/licenses/by/4.0/.

(c) The Author(s) 2020 